\title{
On periodicity of systems of rational difference equations
}

\author{
Alaa E. Hamza ${ }^{a, b, *}$, Naser Alsulami ${ }^{a, *}$ \\ ${ }^{a}$ Department of Mathematics, College of Science, University of Jeddah, Jeddah 21589, Saudi Arabia. \\ ${ }^{b}$ Department of Mathematics, Faculty of Science, Cairo University, Giza, Egypt.
}

\begin{abstract}
In this paper, we investigate the periodicity of two systems of rational sequences of second and third order, respectively. The systems include a permutation that gives the ability of changing the appearance of components of solutions in the equations of the systems. We find periods of systems in terms of the order of the permutation. The periodicity of two more systems of maximum type are studied. Finally, many illustrative examples are given.
\end{abstract}

Keywords: Systems of difference equations, permutation groups, periodicity.

2020 MSC: 39A23.

(C)2022 All rights reserved.

\section{Introduction}

The theory of permutation groups is important to diverse area of mathematics such as Galois theory, invariant theory, the representation theory of Lie groups, and combinatorics. See for instance [1, 8]. In general, the theory of abstract groups plays an important part in present day mathematics and science. Groups arise in a bewildering number of apparently unconnected subjects. Thus they appear in algebra and analysis, in geometry and topology, in crystallography and quantum mechanics, in physics and chemistry, and even in biology, see $[2,16]$. In this paper, as another application of the theory of permutation groups, we investigate the periodicity of systems of difference equations. For the basics of the theory of difference equations, we refer the reader to the monographs [3,10]. Recently, there has been a great interest in difference equations, because they describe naturally many real-life problems in biology, ecology, genetics, psychology, sociology, and so forth. Iricanian and Stevic [9], inspired by the work of Lyness [11-13] who proved that the difference equation

$$
x_{n+1}=\frac{1+x_{n}}{x_{n-1}}, \quad n \in \mathbb{N}_{0}
$$

is periodic with period five, investigated the periodicity of the systems

$$
x_{n+1}^{(1)}=\frac{x_{n}^{(2)}+1}{x_{n-1}^{(3)}}, \quad x_{n+1}^{(2)}=\frac{x_{n}^{(3)}+1}{x_{n-1}^{(4)}}, \ldots x_{n+1}^{(k)}=\frac{x_{n}^{(1)}+1}{x_{n-1}^{(2)}},
$$

\footnotetext{
${ }^{*}$ Corresponding author

Email addresses: hamzaaeg2003@yahoo.com (Alaa E. Hamza), nasir6655@hotmail .com (Naser Alsulami)

doi: $10.22436 /$ jmcs.025.04.08
}

Received: 2021-06-04 Revised: 2021-06-29 Accepted: 2021-07-13 
and

$$
x_{n+1}^{(1)}=\frac{x_{n}^{(2)}+x_{n-1}^{(3)}+1}{x_{n-2}^{(4)}}, x_{n+1}^{(2)}=\frac{x_{n}^{(3)}+x_{n-1}^{(4)}+1}{x_{n-2}^{(5)}}, \ldots x_{n+1}^{(k)}=\frac{x_{n}^{(1)}+x_{n-1}^{(2)}+1}{x_{n-2}^{(3)}},
$$

For some pertinent results about periodicity, we refer the reader to the interesting papers $[4-6,15,17]$.

Motivated by the above results, we investigate the periodicity of the following systems

$$
x_{n+1}^{(1)}=\frac{x_{n}^{\pi(1)}+1}{x_{n-1}^{\pi^{2}(1)}}, x_{n+1}^{(2)}=\frac{x_{n}^{\pi(2)}+1}{x_{n-1}^{\pi^{2}(2)}}, \ldots x_{n+1}^{(k)}=\frac{x_{n}^{\pi(k)}+1}{x_{n-1}^{\pi^{2}(k)}}
$$

and

$$
x_{n+1}^{(1)}=\frac{x_{n}^{\pi(1)}+x_{n-1}^{\pi^{2}(1)}+1}{x_{n-2}^{\pi^{3}(1)}}, x_{n+1}^{(2)}=\frac{x_{n}^{\pi(2)}+x_{n-1}^{\pi^{2}(2)}+1}{x_{n-2}^{\pi^{3}(2)}}, \ldots x_{n+1}^{(k)}=\frac{x_{n}^{\pi(k)}+x_{n-1}^{\pi^{2}(k)}+1}{x_{n-2}^{\pi^{3}(k)}},
$$

where $\pi \in \mathcal{S}_{k}$. Every choice of a permutation $\pi$ gives a system of difference equations. Thus in fact System (1.1) or System (1.2) represents $k$ ! systems. It is well-known that for any permutation $\pi \in S_{k}$, there is a natural number $l$ such that the property $\pi^{l}=I$ holds, where I is the identity permutation and $\pi^{l}$ is the composition of $\pi$ with itself l-times. The smallest $l$ for which this property holds is called the order of $\pi$.

Definition 1.1. The system

$$
\begin{aligned}
x_{n+1}^{(1)}= & f_{1}\left(x_{n}^{\pi(1)}, x_{n-1}^{\pi^{2}(1)}, \ldots, x_{n-s}^{\pi^{s+1}(1)}\right), \\
& \vdots \\
x_{n+1}^{(k)} & =f_{k}\left(x_{n}^{\pi(k)}, x_{n-1}^{\pi^{2}(k)}, \ldots, x_{n-s}^{\pi^{s+1}(k)}\right)
\end{aligned}
$$

is called periodic with period $d$ if every positive solution $\left(x_{n}^{(1)}, x_{n}^{(2)}, \ldots, x_{n}^{(k)}\right)$ satisfies

$$
x_{n+d}^{(i)}=x_{n}^{(i)}, n \in \mathbb{Z}^{\geqslant 0}, i \in \mathbb{Z}_{k} .
$$

A solution $\left(x_{n}^{(1)}, x_{n}^{(2)}, \ldots, x_{n}^{(k)}\right)$ is said to be positive if each component $x_{n}^{i}$ is positive for every $n$.

\section{Main results}

In this section we study the periodicity of systems (1.1) and (1.2).

Theorem 2.1. System (1.1) is periodic with period $\mathrm{l}$ if $\mathrm{l}=0 \bmod 5$ and is periodic with period $5 \mathrm{l}$ if $\mathrm{l} \neq 0$ mod 5 .

Proof. Let $\left(x_{n}^{(1)}, x_{n}^{(2)}, \ldots, x_{n}^{(k)}\right)$ be a positive solution of system (1.1). We can see that

$$
x_{n+d}^{(1)}=x_{n+d-5 r}^{\pi^{5 r}(1)}, n \in \mathbb{Z}^{\geqslant 0}, r \in \mathbb{N}, d \geqslant 5 r .
$$

Indeed, we have

$$
x_{n+d}^{(1)}=\frac{x_{n+d-1}^{\pi(1)}+1}{x_{n+d-2}^{\pi^{2}(1)}}=\frac{\left(\frac{x_{n+d-2}^{\pi^{2}(1)}+1}{x_{n+d-3}^{\pi^{3}(1)}}\right)+1}{x_{n+d-2}^{\pi^{2}(1)}}=\frac{x_{n+d-2}^{\pi^{2}(1)}+1+x_{n+d-3}^{\pi^{3}(1)}}{x_{n+d-3}^{\pi^{3}(1)} x_{n+d-2}^{\pi^{2}(1)}}=\frac{\left(\frac{x_{n+d-3}^{\pi^{3}(1)}+1}{x_{n+d-4}^{\pi^{4}(1)}}\right)+1+x_{n+d-3}^{\pi^{3}(1)}}{x_{n+d-3}^{\pi^{3}(1)}\left(\frac{x_{n+d-3}^{\pi^{3}(1)}}{x_{n+d-4}^{\pi^{4}(1)}}\right)} .
$$

It follows that

$$
x_{n+d}^{(1)}=\frac{x_{n+d-3}^{\pi^{3}(1)}+1+x_{n+d-4}^{\pi^{4}(1)}+x_{n+d-3}^{\pi^{3}(1)} x_{n+d-4}^{\pi^{4}(1)}}{x_{n+d-3}^{\pi^{3}(1)}\left(x_{n+d-3}^{\pi^{3}(1)}+1\right)}
$$




$$
\begin{aligned}
& =\frac{x_{n+d-3}^{\pi^{3}(1)}+1+x_{n+d-4}^{\pi^{4}(1)}\left(1+x_{n+d-3}^{\pi^{3}(1)}\right)}{x_{n+d-3}^{\pi^{3}(1)}\left(x_{n+d-3}^{\pi^{3}(1)}+1\right)} \\
& =\frac{1+x_{n+d-4}^{\pi^{4}(1)}}{x_{n+d-3}^{\pi^{3}(1)}}=x_{n+d-5}^{\pi^{5}(1)} \\
& \vdots \\
& =x_{n+d-5 r}^{\pi^{5 r}(1)}, n \in \mathbb{Z}^{\geqslant 0}, r \in \mathbb{N} .
\end{aligned}
$$

Assume that $l=0 \bmod 5$. Then $l=5 r$ for some $r \in \mathbb{N}$. Setting $d=5 r$ in (2.1), we get $x_{n+l}^{(1)}=x_{n}^{(1)}$. Similarly, $x_{n+l}^{(i)}=x_{n}^{(i)}, i=1, \ldots, k$. Therefore, system (1.1) is periodic with period $l$. For the case $l \neq 0$ mod 5, putting $d=5 l$ and $r=l$ in (2.1), we obtain $x_{n+5 l}^{(1)}=x_{n}^{(1)}$. Similarly, $x_{n+5 l}^{(i)}=x_{n}^{(i)}, i=1, \ldots, k$. Therefore system (1.1) is periodic with period $5 l$.

The following Theorem establishes the periodicity of systems of second-order of maximum type.

Theorem 2.2. The system

$$
x_{n+1}^{(1)}=\frac{\max \left\{x_{n}^{\pi(1)}, 1\right\}}{x_{n-1}^{\pi^{2}(1)}}, x_{n+1}^{(2)}=\frac{\max \left\{x_{n}^{\pi(2)}, 1\right\}}{x_{n-1}^{\pi^{2}(2)}}, \ldots, x_{n+1}^{(k)}=\frac{\max \left\{x_{n}^{\pi(k)}, 1\right\}}{x_{n-1}^{\pi^{2}(k)}},
$$

is periodic with period $l$ if $l=0 \bmod 5$ and is periodic with period $5 l$ if $l \neq 0 \bmod 5$.

Proof. Let $\left(x_{n}^{(1)}, x_{n}^{(2)}, \ldots, x_{n}^{(k)}\right)$ be a positive solution of system (2.2). Then, it satisfies (2.1). Indeed,

$$
\begin{aligned}
x_{n+d}^{(1)}=\frac{\max \left\{x_{n+d-1}^{\pi(1)}, 1\right\}}{x_{n+d-2}^{\pi^{2}(1)}} & =\frac{\max \left\{\max \left\{x_{n+d-2}^{\pi^{2}(1)}, 1\right\}, x_{n+d-3}^{\pi^{3}(1)}\right\}}{x_{n+d-2}^{\pi^{2}(1)} x_{n+d-3}^{\pi^{3}(1)}} \\
& =\frac{\max \left\{x_{n+d-2}^{\pi^{2}(1)}, 1, x_{n+d-3}^{\pi^{3}(1)}\right\}}{x_{n+d-2}^{\pi^{2}(1)} x_{n+d-3}^{\pi^{3}(1)}} \\
& =\frac{\max \left\{\frac{\max \left\{x_{n+d-3}^{\pi^{3}(1)}, 1\right\}}{x_{n+d-4}^{\pi^{4}(1)}}, 1, x_{n+d-3}^{\pi^{3}(1)}\right\}}{\max \left\{x_{n+d-3}^{\pi^{3}(1)}\right\}} \\
& =\frac{\max \left\{\max \left\{x_{n+d-3}^{\pi^{3}(1)}, 1\right\}, x_{n+d-4}^{\pi^{4}(1)}, x_{n+d-3}^{\pi^{3}(1)} x_{n+d-4}^{\pi^{4}(1)}\right\}}{x_{n+d-3}^{\pi^{3}(1)} \max \left\{x_{n+d-3}^{\pi^{3}(1)}, 1\right\}} \\
& =\frac{\max \left\{\max \left\{x_{n+d-3}^{\pi^{3}(1)}, 1\right\}, x_{n+d-4}^{\pi^{4}(1)} \max \left\{x_{n+d-3}^{\pi^{3}(1)}, 1\right\}\right\}}{x_{n+d-3}^{\pi^{3}(1)} \max \left\{x_{n+d-3}^{\pi^{3}(1)}, 1\right\}}
\end{aligned}
$$

It follows that

$$
x_{n+d}^{(1)}=\frac{\max \left\{1, x_{n+d-4}^{\pi^{4}(1)}\right\}}{x_{n+d-3}^{\pi^{3}(1)}}=x_{n+d-5}^{\pi^{5}(1)}=\cdots=x_{n+d-5 r}^{\pi^{5 r}(1)}, n \in \mathbb{Z}^{\geqslant 0}, r \in \mathbb{N}, d \geqslant 5 r .
$$


For the case $l=0 \bmod 5$, there exists $r \in \mathbb{N}$ such that $l=5 r$. Set $d=5 r$ in (2.1) to obtain $x_{n+l}^{(1)}=x_{n}^{(1)}$. Similarly, we obtain $x_{n+l}^{(i)}=x_{n}^{(i)}, i=1, \ldots k$. So system (2.2) is periodic with period $l$.

Now, assume that $l \neq 0$ mod 5. Putting $d=5 l$ and $r=l$ in (2.1), we obtain $x_{n+5 l}^{(1)}=x_{n}^{(1)}$. Similarly, $x_{n+5 l}^{(i)}=x_{n}^{(i)}, i=1, \ldots, k$. Therefore system (2.2) is periodic with period $5 l$.

In the following result, we prove the periodicity of system (1.2), with period $l 2^{3-i}$, under the condition $\operatorname{GCD}(l, 8)=2^{i}, i=0,1,2,3$. Here, $\operatorname{GCD}(a, b)$ is the greatest common divisor of $a$ and $b$.

Theorem 2.3. System (1.2) is periodic with period $12^{3-i}$ if $\operatorname{GCD}(l, 8)=2^{i}, i=0,1,2,3$.

Proof. Let $\left(x_{n}^{(1)}, x_{n}^{(2)}, \ldots, x_{n}^{(k)}\right)$ be a positive solution of system (1.2). We prove

$$
x_{n+d}^{(1)}=x_{n+d-8}^{\pi^{8}(1)}, n \in \mathbb{Z}^{\geqslant 0}, d \geqslant 8 .
$$

We have

$$
\begin{aligned}
& x_{n+d}^{(1)}=\frac{x_{n+d-1}^{\pi(1)}+x_{n+d-2}^{\pi^{2}(1)}+1}{x_{n+d-3}^{\pi^{3}(1)}} \\
& =\frac{\left(\frac{x_{n+d-2}^{\pi^{2}(1)}+x_{n+d-3}^{\pi^{3}(1)}+1}{x_{n+d-4}^{\pi^{4}(1)}}\right)+x_{n+d-2}^{\pi^{2}(1)}+1}{x_{n+d-3}^{\pi^{3}(1)}} \\
& =\frac{x_{n+d-2}^{\pi^{2}(1)}+x_{n+d-3}^{\pi^{3}(1)}+1+x_{n+d-4}^{\pi^{4}(1)} x_{n+d-2}^{\pi^{2}(1)}+x_{n+d-4}^{\pi^{4}(1)}}{x_{n+d-4}^{\pi^{3}(1)} x_{n+d-3}^{\pi^{3}(1)}} \\
& =\frac{\left(x_{n+d-2}^{\pi^{2}(1)}+1\right)\left(x_{n+d-4}^{\pi^{4}(1)}+1\right)+x_{n+d-3}^{\pi^{3}(1)}}{x_{n+d-3}^{\pi^{3}(1)} x_{n+d-4}^{\pi^{4}(1)}} \\
& =\frac{\left(\frac{x_{n+d-3}^{\pi^{3}(1)}+x_{n+d-4}^{\pi^{4}(1)}+1}{x_{n+d-5}^{\pi^{5}(1)}}+1\right)\left(x_{n+d-4}^{\pi^{4}(1)}+1\right)+x_{n+d-3}^{\pi^{3}(1)}}{x_{n+d-3}^{\pi^{3}(1)} x_{n+d-4}^{\pi^{4}(1)}} \\
& =\frac{\left(x_{n+d-3}^{\pi^{3}(1)}+x_{n+d-4}^{\pi^{4}(1)}+1+x_{n+d-5}^{\pi^{5}(1)}\right)\left(x_{n+d-4}^{\pi^{4}(1)}+1\right)+x_{n+d-5}^{\pi^{5}(1)} x_{n+d-3}^{\pi^{3}(1)}}{x_{n+d-3}^{\pi^{3}(1)} x_{n+d-4}^{\pi^{4}(1)} x_{n+d-5}^{\pi^{5}(1)}}
\end{aligned}
$$

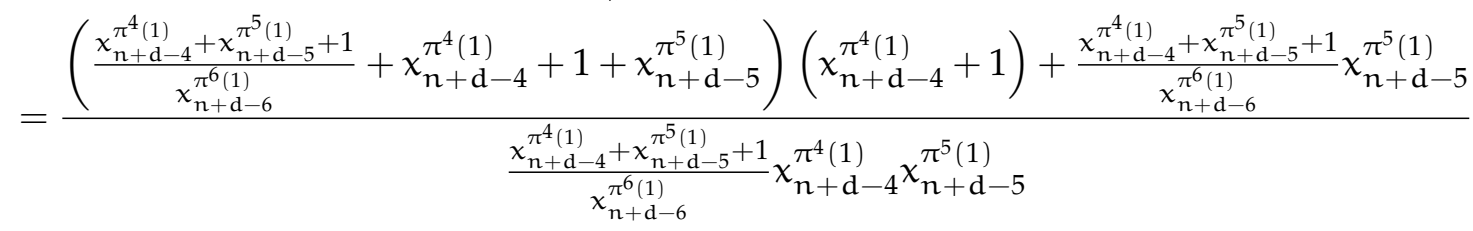

$$
\begin{aligned}
& =\frac{\left(x_{n+d-6}^{\pi^{6}(1)}+1\right)\left(x_{n+d-4}^{\pi^{4}(1)}+1\right)+x_{n+d-5}^{\pi^{5}(1)}}{x_{n+d-4}^{\pi^{4}(1)} x_{n+d-5}^{\pi^{5}(1)}} . \\
& =\frac{\left(x_{n+d-6}^{\pi^{6}(1)}+1\right)\left(\frac{x_{n+d-5}^{\pi^{5}(1)}+x_{n+d-6}^{\pi^{6}(1)}+1}{x_{n+d-7}^{\pi^{7}(1)}}+1\right)+x_{n+d-5}^{\pi^{5}(1)}}{\left(\frac{x_{n+d-5}^{\pi^{5}(1)}+x_{n+d-6}^{\pi^{6}(1)}+1}{x_{n+d-7}^{\pi^{7}(1)}}\right) x_{n+d-5}^{\pi^{5}(1)}} .
\end{aligned}
$$


This implies that

$$
\begin{aligned}
& x_{n+d}^{(1)}=\frac{\left(x_{n+d-5}^{\pi^{5}(1)}+x_{n+d-6}^{\pi^{6}(1)}+1+x_{n+d-7}^{\pi^{7}(1)}\right)\left(x_{n+d-6}^{\pi^{6}(1)}+1\right)+x_{n+d-5}^{\pi^{5}(1)} x_{n+d-7}^{\pi^{7}(1)}}{\left(x_{n+d-5}^{\pi^{5}(1)}+x_{n+d-6}^{\pi^{6}(1)}+1\right) x_{n+d-5}^{\pi^{5}(1)}} \\
& =\frac{\left(x_{n+d-6}^{\pi^{6}(1)}+1\right)\left(\frac{x_{n+d-6}^{\pi^{6}(1)}+x_{n+d-7}^{\pi^{7}(1)}+1}{x_{n+d-8}^{\pi^{8}(1)}}+x_{n+d-6}^{\pi^{6}(1)}+1+x_{n+d-7}^{\pi^{7}(1)}\right)+\frac{x_{n+d-6}^{\pi^{6}(1)}+x_{n+d-7}^{\pi^{7}(1)}}{x_{n+d-8}^{\pi^{8}(1)}} x_{n+d-7}^{\pi^{7}(1)}}{\left(\frac{x_{n+d-6}^{\pi^{6}(1)}+x_{n+d-7}^{\pi^{7}(1)}+1}{x_{n+d-8}^{\pi^{8}(1)}}+x_{n+d-6}^{\pi^{6}(1)}+1\right) x_{n+d-5}^{\pi^{5}(1)}} \\
& =\frac{x_{n+d-6}^{\pi^{6}(1)}+x_{n+d-7}^{\pi^{7}(1)}+1}{x_{n+d-8}^{\pi^{8}(1)}} \frac{\left[\left(x_{n+d-6}^{\pi^{6}(1)}+1\right)\left(x_{n+d-8}^{\pi^{8}(1)}+1\right)+x_{n+d-7}^{\pi^{7}(1)}\right]}{\left(\frac{x_{n+d-6}^{\pi^{6}(1)}+x_{n+d-7}^{\pi^{7}(1)}+1}{x_{n+d-8}^{\pi^{8}(1)}}+x_{n+d-6}^{\pi^{6}(1)}+1\right) x_{n+d-5}^{\pi^{5}(1)}} \\
& =\frac{x_{n+d-6}^{\pi^{6}(1)}+x_{n+d-7}^{\pi^{7}(1)}+1}{x_{n+d-5}^{\pi^{5}(1)}}=x_{n+d-8}^{\pi^{8}(1)} .
\end{aligned}
$$

We begin by the first possibility $i=0$, that is $\operatorname{GCD}(l, 8)=1$. Then $l$ has the form $l=2 r-1$ for some $r \in \mathbb{N}$. Setting $d=8 l$ in (2.3), we obtain

$$
x_{n+8 l}^{(1)}=x_{n+8 l-8}^{\pi^{8}(1)}=x_{n+8 l-8 \times 3}^{\pi^{8 \times 3}(1)}=\cdots=x_{n+8 l-8 l}^{\pi^{8 l}(1)}=x_{n}^{(1)}, n \in \mathbb{Z}^{\geqslant 0} .
$$

So, $x_{n+8 l}^{(1)}=x_{n}^{(1)}$. Similarly, $x_{n+8 l}^{(j)}=x_{n}^{(j)}, j=1,2, \ldots, k$. Therefore system (1.2) is periodic with period $8 \mathrm{l}$.

The second possibility $i=1$ means that $\operatorname{GCD}(l, 8)=2$. Then in this case $l$ has the form $l=4 r-2$ for some $r \in \mathbb{N}$. Use (2.3) with $d=4 l$ to conclude that

$$
x_{n+4 l}^{(1)}=x_{n+4 l-8}^{\pi^{8}(1)}=x_{n+4 l-4 \times 2}^{\pi^{4 \times 2}(1)}=x_{n+4 l-4 \times 6}^{\pi^{4 \times 6}(1)}=\cdots=x_{n+4 l-4(4 r-2)}^{\pi^{4 \times(4 r-2)}(1)}=x_{n+4 l-4 l}^{\pi^{4 l}(1)}=x_{n}^{(1)} .
$$

Similarly, we can see that $x_{n+4 l}^{(j)}=x_{n}^{(j)}, j=1,2, \ldots, k$. Therefore system (1.2) is periodic with period $4 l$. Consider $i=2$, i.e., $\operatorname{GCD}(l, 8)=4$. Thus $l$ has the form $l=8 r-4$ for some $r \in \mathbb{N}$. Use (2.3) with $d=2 l$ to obtain

$$
x_{n+2 l}^{(1)}=x_{n+2 l-8}^{\pi^{8}(1)}=x_{n+2 l-2 \times 4}^{\pi^{2 \times 4}(1)}=x_{n+2 l-2 \times 12}^{\pi^{2 \times 12}(1)}=\cdots=x_{n+2 l-2 \times(8 r-4)}^{\pi^{2 \times(8 r-4)}(1)}=x_{n+2 l-2 l}^{\pi^{2 l}(1)}=x_{n}^{(1)} .
$$

Similarly we can see that $x_{\mathfrak{n}+2 l}^{(j)}=x_{\mathfrak{n}}^{(j)}, \mathfrak{j}=1,2, \ldots, k$. Therefore the system (1.2) is periodic with period $2 l$.

Finally, for $i=3, \operatorname{GCD}(l, 8)=8$ and consequently $l=8 r$ for some $r \in \mathbb{N}$. Use (2.3) with $d=l$ to conclude that

$$
x_{n+l}^{(1)}=x_{n+l-8}^{\pi^{8}(1)}=x_{n+l-8 \times 2}^{\pi^{8 \times 2}(1)}=\cdots=x_{n+l-8 r}^{\pi^{8 r}(1)}=x_{n+l-l}^{\pi^{l}(1)}=x_{n}^{(1)}
$$

so, $x_{n+l}^{(1)}=x_{n}^{(1)}$. Same calculations show that $x_{n+l}^{(j)}=x_{n}^{(j)}, j=1,2, \ldots, k$. Therefore system (1.2) is periodic with period $l$.

Theorem 2.4. The system

$$
x_{n+1}^{(1)}=\frac{\max \left\{x_{n}^{\pi(1)}, x_{n-1}^{\pi^{2}(1)}, 1\right\}}{x_{n-2}^{\pi^{3}(1)}}, x_{n+1}^{(2)}=\frac{\max \left\{x_{n}^{\pi(2)}, x_{n-1}^{\pi^{2}(2)}, 1\right\}}{x_{n-2}^{\pi^{3}(2)}}, \ldots x_{n+1}^{(k)}=\frac{\max \left\{x_{n}^{\pi(k)}, x_{n-1}^{\pi^{2}(k)}, 1\right\}}{x_{n-2}^{\pi^{3}(k)}},
$$

is periodic with period $12^{3-i}$ if $\operatorname{GCD}(l, 8)=2^{i}, i=0,1,2,3$. 
Proof. Let $\left(x_{n}^{(1)}, \chi_{n}^{(2)}, \ldots, x_{n}^{(k)}\right)$ be a positive solution of system (2.4). We can show (2.3). Indeed,

$$
\begin{aligned}
x_{n+d}^{(1)}= & \frac{\max \left\{\max \left\{x_{n+d-3^{\pi^{3}(1)}}, x_{n+d-4}^{\pi^{4}(1)}, 1\right\}, x_{n+d-4}^{\pi^{4}(1)} \max \left\{x_{n+d-3}^{\pi^{3}(1)}, x_{n+d-4}^{\pi^{4}(1)}, 1\right\}, x_{n+d-5}^{\pi^{5}(1)} \max \left\{x_{n+d-3}^{\pi^{3}(1)}, x_{n+d-4}^{\pi^{4}(1)}, 1\right\}\right\}}{x_{n+d-5}^{\pi^{5}(1)} x_{n+d-4}^{\pi^{4}(1)} x_{n+d-3}^{\pi^{3}(1)}} \\
= & \frac{\max \left\{x_{n+d-3^{2}}^{\pi^{3}(1)}, x_{n+d-4}^{\pi^{4}(1)}, 1\right\} \max \left\{x_{n+d-4}^{\pi^{4}(1)}, x_{n+d-5}^{\pi^{5}(1)}, 1\right\}}{x_{n+d-5}^{\pi^{5}(1)} x_{n+d-4}^{\pi^{4}(1)} x_{n+d-3}^{\pi^{3}(1)}} \\
= & \frac{\max \left\{\frac{\max \left\{x_{n+d-4}^{\pi^{4}(1)}, x_{n+d-5}^{\pi^{5}(1)}, 1\right\}}{\pi_{n+d-6}^{\pi^{6}(1)}}, x_{n+d-4}^{\pi^{4}(1)}, 1\right\} \max \left\{x_{n+d-4}^{\pi^{4}(1)}, x_{n+d-5}^{\pi^{5}(1)}, 1\right\}}{x_{n+d-5}^{\pi^{5}(1)} x_{n+d-4}^{\pi^{4}(1)} \frac{\max \left\{x_{n+d-4}^{\pi^{4}(1)}, x_{n+d-5}^{\pi^{5}(1)}\right\}}{x_{n+d-6}^{\pi^{6}(1)}}} \\
= & \frac{\max \left\{\max \left\{x_{n+d-4}^{\pi^{4}(1)} x_{n+d-5}^{\pi^{5}(1)}, 1\right\}, x_{n+d-6}^{\pi^{6}(1)} x_{n+d-4}^{\pi^{4}(1)}, x_{n+d-6}^{\pi^{6}(1)}\right\} \max \left\{x_{n+d-4}^{\pi^{4}(1)}, x_{n+d-5}^{\pi^{5}(1)}, 1\right\}}{x_{n+d-5}^{\pi^{5}(1)} x_{n+d-4}^{\pi^{4}(1)} \max \left\{x_{n+d-4}^{\pi^{4}(1)}, x_{n+d-5}^{\pi^{5}(1)}, 1\right\}} .
\end{aligned}
$$

We deduce that

$$
\begin{aligned}
x_{n+d}^{(1)} & =\frac{\max \left\{x_{n+d-4}^{\pi^{4}(1)}, x_{n+d-5}^{\pi^{5}(1)}, 1, x_{n+d-6}^{\pi^{6}(1)} x_{n+d-4}^{\pi^{4}(1)}, x_{n+d-6}^{\pi^{6}(1)}\right\}}{x_{n+d-5}^{\pi^{5}(1)} x_{n+d-4}^{\pi^{4}(1)}} \\
= & \frac{\max \left\{\frac{\max \left\{x_{n+d-5}^{\pi^{5}(1)}, x_{n+d-6}^{\pi^{6}(1)}\right\}}{x_{n+d-7}^{\pi^{7}(1)}}, x_{n+d-5}^{\pi^{5}(1)}, 1, x_{n+d-6}^{\pi^{6}(1)} \frac{\max \left\{x_{n+d-5}^{\pi^{5}(1)}, x_{n+d-6}^{\pi^{6}(1)}\right\}}{x_{n+d-7}^{\pi^{7}(1)}}, x_{n+d-6}^{\pi^{6}(1)}\right\}}{x_{n+d-5}^{\pi^{5}(1)} \frac{\max \left\{x_{n+d-5}^{\pi^{5}(1)}, \pi_{n+d-6}^{\pi^{6}(1)}, 1\right\}}{x_{n+d-7}^{\pi^{7}(1)}}} \\
= & \frac{\max \left\{\max \left\{x_{n+d-5}^{\pi^{5}(1)}, x_{n+d-6}^{\pi^{6}(1)}, 1\right\}, x_{n+d-7}^{\pi^{7}(1)} x_{n+d-5}^{\pi^{5}(1)}, x_{n+d-7}^{\pi^{7}(1)}, x_{n+d-6}^{\pi^{6}(1)} \max \left\{x_{n+d-5}^{\pi^{5}(1)}, x_{n+d-6}^{\pi^{6}(1)}, 1\right\}, x_{n+d-7}^{\pi^{7}(1)} x_{n+d-6}^{\pi^{6}(1)}\right\}}{x_{n+d-5}^{\pi^{5}(1)} \max \left\{x_{n+d-5}^{\pi^{5}(1)}, x_{n+d-6}^{\pi^{6}(1)}, 1\right\}} \\
= & \frac{\max \left\{\max \left\{x_{n+d-5}^{\pi^{5}(1)}, x_{n+d-6}^{\pi^{6}(1)}, 1\right\}, x_{n+d-7}^{\pi^{7}(1)} \max \left\{x_{n+d-5}^{\pi^{5}(1)}, x_{n+d-6}^{\pi^{6}(1)}, 1\right\}, x_{n+d-6}^{\pi^{6}(1)} \max \left\{x_{n+d-5}^{\pi^{5}(1)}, x_{n+d-6}^{\pi^{6}(1)}, 1\right\}\right\}}{x_{n+d-5}^{\pi^{5}(1)} \max \left\{x_{n+d-5}^{\pi^{5}(1)}, x_{n+d-6}^{\pi^{6}(1)}, 1\right\}} \\
= & \frac{\max \left\{x_{n+d-6}^{\pi^{6}(1)}, x_{n+d-7}^{\pi^{\pi^{7}}(1)}, 1\right\}}{x_{n+d-5}^{\pi^{5}(1)}}=x_{n+d-8}^{\pi^{8}(1)}
\end{aligned}
$$

The rest of the proof follows similarly as in proof of Theorem 2.3.

\section{Illustrative examples}

(i) The systems

$$
\begin{aligned}
& x_{n+1}^{(1)}=\frac{x_{n}^{(5)}+1}{x_{n-1}^{(3)}}, x_{n+1}^{(2)}=\frac{x_{n}^{(7)}+1}{x_{n-1}^{(4)}}, x_{n+1}^{(3)}=\frac{x_{n}^{(1)}+1}{x_{n-1}^{(5)}}, x_{n+1}^{(4)}=\frac{x_{n}^{(8)}+1}{x_{n-1}^{(2)}}, x_{n+1}^{(5)}=\frac{x_{n}^{(3)}+1}{x_{n-1}^{(1)}}, \\
& x_{n+1}^{(6)}=\frac{x_{n}^{(10)}+1}{x_{n-1}^{(9)}}, x_{n+1}^{(7)}=\frac{x_{n}^{(4)}+1}{x_{n-1}^{(8)}}, x_{n+1}^{(8)}=\frac{x_{n}^{(2)}+1}{x_{n-1}^{(7)}}, x_{n+1}^{(9)}=\frac{x_{n}^{(6)}+1}{x_{n-1}^{(10)}}, x_{n+1}^{(10)}=\frac{x_{n}^{(9)}+1}{x_{n-1}^{(6)}}
\end{aligned}
$$


and

$$
\begin{aligned}
& x_{n+1}^{(1)}=\frac{\max \left\{x_{n}^{(5)}, 1\right\}}{x_{n-1}^{(3)}}, x_{n+1}^{(2)}=\frac{\max \left\{x_{n}^{(7)}, 1\right\}}{x_{n-1}^{(4)}}, x_{n+1}^{(3)}=\frac{\max \left\{x_{n}^{(1)}, 1\right\}}{x_{n-1}^{(5)}}, \\
& x_{n+1}^{(4)}=\frac{\max \left\{x_{n}^{(8)}, 1\right\}}{x_{n-1}^{(2)}}, x_{n+1}^{(5)}=\frac{\max \left\{x_{n}^{(3)}, 1\right\}}{x_{n-1}^{(1)}}, x_{n+1}^{(6)}=\frac{\max \left\{x_{n}^{(10)}, 1\right\}}{x_{n-1}^{(9)}}, \\
& x_{n+1}^{(7)}=\frac{\max \left\{x_{n}^{(4)}, 1\right\}}{x_{n-1}^{(8)}}, x_{n+1}^{(8)}=\frac{\max \left\{x_{n}^{(2)}+1\right\}}{x_{n-1}^{(7)}}, x_{n+1}^{(9)}=\frac{\max \left\{x_{n}^{(6)}+1\right\}}{x_{n-1}^{(10)}}, x_{n+1}^{(10)}=\frac{\max \left\{x_{n}^{(9)}+1\right\}}{x_{n-1}^{(6)}} .
\end{aligned}
$$

are periodic with period 60. The permutation which corresponds to each of these systems is $\pi=$

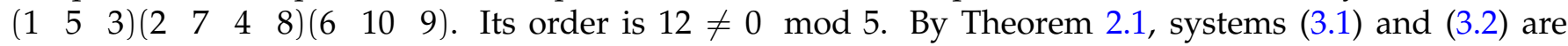
periodic with period 60 .

(ii) The systems

$$
\begin{aligned}
& x_{n+1}^{(1)}=\frac{x_{n}^{(5)}+x_{n-1}^{(9)}+1}{x_{n-2}^{(3)}}, x_{n+1}^{(2)}=\frac{x_{n}^{(12)}+x_{n-1}^{(8)}+1}{x_{n-2}^{(10)}}, x_{n+1}^{(3)}=\frac{x_{n}^{(4)}+x_{n-1}^{(7)}+1}{x_{n-2}^{(11)}}, \\
& x_{n+1}^{(4)}=\frac{x_{n}^{(7)}+x_{n-1}^{(11)}+1}{x_{n-2}^{(2)}}, x_{n+1}^{(5)}=\frac{x_{n}^{(9)}+x_{n-1}^{(3)}+1}{x_{n-2}^{(4)}}, x_{n+1}^{(6)}=\frac{x_{n}^{(1)}+x_{n-1}^{(5)}+1}{x_{n-2}^{(9)}}, \\
& x_{n+1}^{(7)}=\frac{x_{n}^{(11)}+x_{n-1}^{(2)}+1}{x_{n-2}^{(12)}}, x_{n+1}^{(8)}=\frac{x_{n}^{(10)}+x_{n-1}^{(6)}+1}{x_{n-2}^{(1)}}, x_{n+1}^{(9)}=\frac{x_{n}^{(3)}+x_{n-1}^{(4)}+1}{x_{n-2}^{(7)}}, \\
& x_{n+1}^{(10)}=\frac{x_{n}^{(6)}+x_{n-1}^{(1)}+1}{x_{n-2}^{(5)}}, x_{n+1}^{(11)}=\frac{x_{n}^{(2)}+x_{n-1}^{(12)}+1}{x_{n-2}^{(8)}}, x_{n+1}^{(12)}=\frac{x_{n}^{(8)}+x_{n-1}^{(10)}+1}{x_{n-2}^{(6)}},
\end{aligned}
$$

and

$$
\begin{aligned}
& x_{n+1}^{(1)}=\frac{\max \left\{x_{n}^{(5)}+x_{n-1}^{(9)}, 1\right\}}{x_{n-2}^{(3)}}, x_{n+1}^{(2)}=\frac{\max \left\{x_{n}^{(12)}+x_{n-1}^{(8)}, 1\right\}}{x_{n-2}^{(10)}}, x_{n+1}^{(3)}=\frac{x_{n}^{(4)}+x_{n-1}^{(7)}+1}{x_{n-2}^{(11)}}, \\
& x_{n+1}^{(4)}=\frac{\max \left\{x_{n}^{(7)}+x_{n-1}^{(11)}, 1\right\}}{x_{n-2}^{(2)}}, x_{n+1}^{(5)}=\frac{\max \left\{x_{n}^{(9)}+x_{n-1}^{(3)}, 1\right\}}{x_{n-2}^{(4)}}, x_{n+1}^{(6)}=\frac{\max \left\{x_{n}^{(1)}+x_{n-1}^{(5)}, 1\right\}}{x_{n-2}^{(9)}}, \\
& x_{n+1}^{(7)}=\frac{\max \left\{x_{n}^{(11)}+x_{n-1}^{(2)}, 1\right\}}{x_{n-2}^{(12)}}, x_{n+1}^{(8)}=\frac{\max \left\{x_{n}^{(10)}+x_{n-1}^{(6)}, 1\right\}}{x_{n-2}^{(1)}}, x_{n+1}^{(9)}=\frac{\max \left\{x_{n}^{(3)}+x_{n-1}^{(4)}, 1\right\}}{x_{n-2}^{(7)}}, \\
& x_{n+1}^{(10)}=\frac{\max \left\{x_{n}^{(6)}+x_{n-1}^{(1)}, 1\right\}}{x_{n-2}^{(5)}}, x_{n+1}^{(11)}=\frac{\max \left\{x_{n}^{(2)}+x_{n-1}^{(12)}, 1\right\}}{x_{n-2}^{(8)}}, x_{n+1}^{(12)}=\frac{\max \left\{x_{n}^{(8)}+x_{n-1}^{(10)}, 1\right\}}{x_{n-2}^{(6)}},
\end{aligned}
$$

are periodic with period 24. The permutation which corresponds to each of these systems is $\pi=$ $\left(\begin{array}{llllllllllll}1 & 5 & 9 & 3 & 4 & 7 & 11 & 2 & 12 & 8 & 10 & 6\end{array}\right)$. Its order is 12 . This implies that systems (3.3) and (3.4) are periodic with period 24 .

(iii) The systems

$$
x_{n+1}^{(1)}=\frac{x_{n}^{(2)}+1}{x_{n-1}^{(3)}}, x_{n+1}^{(2)}=\frac{x_{n}^{(3)}+1}{x_{n-1}^{(5)}}, x_{n+1}^{(3)}=\frac{x_{n}^{(5)}+1}{x_{n-1}^{(4)}}, x_{n+1}^{(4)}=\frac{x_{n}^{(1)}+1}{x_{n-1}^{(2)}}, x_{n+1}^{(5)}=\frac{x_{n}^{(4)}+1}{x_{n-1}^{(1)}},
$$


and

$$
\begin{aligned}
& x_{n+1}^{(1)}=\frac{\max \left\{x_{n}^{(2)}, 1\right\}}{x_{n-1}^{(3)}}, x_{n+1}^{(2)}=\frac{\max \left\{x_{n}^{(3)}, 1\right\}}{x_{n-1}^{(5)}}, \\
& x_{n+1}^{(3)}=\frac{\max \left\{x_{n}^{(5)}, 1\right\}}{x_{n-1}^{(4)}}, x_{n+1}^{(4)}=\frac{\max \left\{x_{n}^{(1)}, 1\right\}}{x_{n-1}^{(2)}}, x_{n+1}^{(5)}=\frac{\max \left\{x_{n}^{(4)}, 1\right\}}{x_{n-1}^{(1)}},
\end{aligned}
$$

are periodic with period 5. Indeed, the permutation which corresponds to each of these systems is $\pi=\left(\begin{array}{lllll}1 & 2 & 3 & 5 & 4\end{array}\right)$. So, its order is 5. This implies that systems (3.5) and (3.6) are periodic with period 5.

(iv) The systems

$$
x_{n+1}^{(1)}=\frac{x_{n}^{(3)}+1}{x_{n-1}^{(2)}}, x_{n+1}^{(2)}=\frac{x_{n}^{(1)}+1}{x_{n-1}^{(3)}}, x_{n+1}^{(3)}=\frac{x_{n}^{(2)}+1}{x_{n-1}^{(1)}}, x_{n+1}^{(4)}=\frac{x_{n}^{(4)}+1}{x_{n-1}^{(4)}}, x_{n+1}^{(5)}=\frac{x_{n}^{(5)}+1}{x_{n-1}^{(5)}},
$$

and

$$
\begin{aligned}
x_{n+1}^{(1)} & =\frac{\max \left\{x_{n}^{(3)}, 1\right\}}{x_{n-1}^{(2)}}, x_{n+1}^{(2)}=\frac{\max \left\{x_{n}^{(1)}, 1\right\}}{x_{n-1}^{(3)}}, \\
x_{n+1}^{(3)} & =\frac{\max \left\{x_{n}^{(2)}, 1\right\}}{x_{n-1}^{(1)}}, x_{n+1}^{(4)}=\frac{\max \left\{x_{n}^{(4)}, 1\right\}}{x_{n-1}^{(4)}}, x_{n+1}^{(5)}=\frac{\max \left\{x_{n}^{(5)}, 1\right\}}{x_{n-1}^{(5)}},
\end{aligned}
$$

are periodic with period 15. The permutation which corresponds to each of these systems is $\pi=$ $\left(\begin{array}{lll}1 & 3 & 2\end{array}\right)(4)(5)$. Its order is $3 \neq 0 \bmod 5$. By Theorem 2.1, systems (3.7) and (3.8) are periodic with period 15.

(v) The systems

$$
\begin{aligned}
& x_{n+1}^{(1)}=\frac{x_{n}^{(3)}+1}{x_{n-1}^{(2)}}, x_{n+1}^{(2)}=\frac{x_{n}^{(4)}+1}{x_{n-1}^{(5)}}, x_{n+1}^{(3)}=\frac{x_{n}^{(2)}+1}{x_{n-1}^{(4)}}, \\
& x_{n+1}^{(4)}=\frac{x_{n}^{(5)}+1}{x_{n-1}^{(1)}}, x_{n+1}^{(5)}=\frac{x_{n}^{(1)}+1}{x_{n-1}^{(3)}}, x_{n+1}^{(6)}=\frac{x_{n}^{(7)}+1}{x_{n-1}^{(6)}}, x_{n+1}^{(7)}=\frac{x_{n}^{(6)}+1}{x_{n-1}^{(7)}},
\end{aligned}
$$

and

$$
\begin{aligned}
& x_{n+1}^{(1)}=\frac{\max \left\{x_{n}^{(3)}, 1\right\}}{x_{n-1}^{(2)}}, x_{n+1}^{(2)}=\frac{\max \left\{x_{n}^{(4)}, 1\right\}}{x_{n-1}^{(5)}}, x_{n+1}^{(3)}=\frac{\max \left\{x_{n}^{(2)}, 1\right\}}{x_{n-1}^{(4)}}, \\
& x_{n+1}^{(4)}=\frac{\max \left\{x_{n}^{(5)}, 1\right\}}{x_{n-1}^{(1)}}, x_{n+1}^{(5)}=\frac{\max \left\{x_{n}^{(1)}, 1\right\}}{x_{n-1}^{(3)}}, x_{n+1}^{(6)}=\frac{\max \left\{x_{n}^{(7)}, 1\right\}}{x_{n-1}^{(6)}}, x_{n+1}^{(7)}=\frac{\max \left\{x_{n}^{(6)}, 1\right\}}{x_{n-1}^{(7)}},
\end{aligned}
$$

are periodic with period 10. The permutation which corresponds to each of these systems is $\pi=$ $\left(\begin{array}{lllll}1 & 3 & 2 & 4 & 5\end{array}\right)\left(\begin{array}{ll}6 & 7\end{array}\right)$. Its order is $10=0 \mathrm{mod}$ 5. By Theorem 2.1, systems (3.9) and (3.10) are periodic with period 10 .

(vi) The systems

$$
x_{n+1}^{(1)}=\frac{x_{n}^{(3)}+x_{n-1}^{(2)}+1}{x_{n-2}^{(1)}}, x_{n+1}^{(2)}=\frac{x_{n}^{(1)}+x_{n-1}^{(3)}+1}{x_{n-2}^{(2)}}, x_{n+1}^{(3)}=\frac{x_{n}^{(2)}+x_{n-1}^{(1)}+1}{x_{n-2}^{(3)}}, x_{n+1}^{(4)}=\frac{x_{n}^{(4)}+x_{n-1}^{(4)}+1}{x_{n-2}^{(4)}}
$$


and

$$
\begin{aligned}
& x_{n+1}^{(1)}=\frac{\max \left\{x_{n}^{(3)}, x_{n-1}^{(2)}, 1\right\}}{x_{n-2}^{(1)}}, x_{n+1}^{(2)}=\frac{\max \left\{x_{n}^{(1)}, x_{n-1}^{(3)}, 1\right\}}{x_{n-2}^{(2)}}, \\
& x_{n+1}^{(3)}=\frac{\max \left\{x_{n}^{(2)}, x_{n-1}^{(1)}, 1\right\}}{x_{n-2}^{(3)}}, x_{n+1}^{(4)}=\frac{\max \left\{x_{n}^{(4)}, x_{n-1}^{(4)}, 1\right\}}{x_{n-2}^{(4)}},
\end{aligned}
$$

are periodic with period 24. The permutation which corresponds to each of these systems is $\pi=$ $\left(\begin{array}{lll}1 & 3 & 2\end{array}\right)(4)$. Its order is 3. This implies that systems (3.11) and (3.12) are periodic with period 24.

(vii) The systems

$$
\begin{aligned}
& x_{n+1}^{(1)}=\frac{x_{n}^{(2)}+x_{n-1}^{(1)}+1}{x_{n-2}^{(2)}}, x_{n+1}^{(2)}=\frac{x_{n}^{(1)}+x_{n-1}^{(2)}+1}{x_{n-2}^{(1)}}, \\
& x_{n+1}^{(3)}=\frac{x_{n}^{(4)}+x_{n-1}^{(3)}+1}{x_{n-2}^{(4)}}, x_{n+1}^{(4)}=\frac{x_{n}^{(3)}+x_{n-1}^{(4)}+1}{x_{n-2}^{(3)}}, x_{n+1}^{(5)}=\frac{x_{n}^{(5)}+x_{n-1}^{(5)}+1}{x_{n-2}^{(5)}},
\end{aligned}
$$

and

$$
\begin{aligned}
& x_{n+1}^{(1)}=\frac{\max \left\{x_{n}^{(2)}, x_{n-1}^{(1)}, 1\right\}}{x_{n-2}^{(2)}}, x_{n+1}^{(2)}=\frac{\max \left\{x_{n}^{(1)}, x_{n-1}^{(2)}, 1\right\}}{x_{n-2}^{(1)}}, \\
& x_{n+1}^{(3)}=\frac{\max \left\{x_{n}^{(4)}, x_{n-1}^{(3)}, 1\right\}}{x_{n-2}^{(4)}}, x_{n+1}^{(4)}=\frac{\max \left\{x_{n}^{(3)}, x_{n-1}^{(4)}, 1\right\}}{x_{n-2}^{(3)}}, x_{n+1}^{(5)}=\frac{\max \left\{x_{n}^{(5)}, x_{n-1}^{(5)}, 1\right\}}{x_{n-2}^{(5)}},
\end{aligned}
$$

are periodic with period 8 . The permutation which corresponds to each of these systems is $\pi=\left(\begin{array}{lll}1 & 2\end{array}\right)\left(\begin{array}{ll}3 & 4\end{array}\right)(5)$. Its order is 2. This implies that systems (3.13) and (3.14) are periodic with period 8.

(viii) The systems

$$
\begin{aligned}
& x_{n+1}^{(1)}=\frac{x_{n}^{(2)}+x_{n-1}^{(3)}+1}{x_{n-2}^{(4)}}, x_{n+1}^{(2)}=\frac{x_{n}^{(3)}+x_{n-1}^{(4)}+1}{x_{n-2}^{(1)}}, \\
& x_{n+1}^{(3)}=\frac{x_{n}^{(4)}+x_{n-1}^{(1)}+1}{x_{n-2}^{(2)}}, x_{n+1}^{(4)}=\frac{x_{n}^{(1)}+x_{n-1}^{(2)}+1}{x_{n-2}^{(3)}}, x_{n+1}^{(5)}=\frac{x_{n}^{(5)}+x_{n-1}^{(5)}+1}{x_{n-2}^{(5)}},
\end{aligned}
$$

and

$$
\begin{aligned}
& x_{n+1}^{(1)}=\frac{\max \left\{x_{n}^{(2)}, x_{n-1}^{(3)}, 1\right\}}{x_{n-2}^{(4)}}, x_{n+1}^{(2)}=\frac{\max \left\{x_{n}^{(3)}, x_{n-1}^{(4)}, 1\right\}}{x_{n-2}^{(1)}}, \\
& x_{n+1}^{(3)}=\frac{\max \left\{x_{n}^{(4)}, x_{n-1}^{(1)}, 1\right\}}{x_{n-2}^{(2)}}, x_{n+1}^{(4)}=\frac{\max \left\{x_{n}^{(1)}, x_{n-1}^{(2)}, 1\right\}}{x_{n-2}^{(3)}}, x_{n+1}^{(5)}=\frac{\max \left\{x_{n}^{(5)}, x_{n-1}^{(5)}, 1\right\}}{x_{n-2}^{(5)}},
\end{aligned}
$$

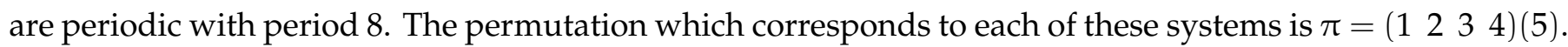
Its order is 4. This implies that systems (3.15) and (3.16) are periodic with period 8. 


\section{Conclusion}

We extended the periodicity results of Iricanian and Stevic [9] to the systems

$$
x_{n+1}^{(1)}=\frac{x_{n}^{\pi(1)}+1}{x_{n-1}^{\pi^{2}(1)}}, \quad x_{n+1}^{(2)}=\frac{x_{n}^{\pi(2)}+1}{x_{n-1}^{\pi^{2}(2)}}, \ldots, x_{n+1}^{(k)}=\frac{x_{n}^{\pi(k)}+1}{x_{n-1}^{\pi^{2}(k)}},
$$

and

$$
x_{n+1}^{(1)}=\frac{x_{n}^{\pi(1)}+x_{n-1}^{\pi^{2}(1)}+1}{x_{n-2}^{\pi^{3}(1)}}, x_{n+1}^{(2)}=\frac{x_{n}^{\pi(2)}+x_{n-1}^{\pi^{2}(2)}+1}{x_{n-2}^{\pi^{3}(2)}}, \ldots x_{n+1}^{(k)}=\frac{x_{n}^{\pi(k)}+x_{n-1}^{\pi^{2}(k)}+1}{x_{n-2}^{\pi^{3}(k)}},
$$

where $\pi \in \mathcal{S}_{k}$. Every choice of a permutation $\pi$ gives a system of difference equations.

\section{Acknowledgment}

The authors would like to thank the anonymous referee and the handling Editor for any useful comments and suggestions, leading to an improvement of the presentation of this article. The authors also would like to thank the Department of Mathematics, University of Jeddah for the encouragement and support.

\section{References}

[1] O. Bogopolski, Introduction to group theory, Cambridge University Press, (1994). 1

[2] F. A. Cotton, Chemical applications of group theory, Third Edition, John Wiley \& Sons, New York, (1990). 1

[3] S. Elaydi, An introduction to difference equations, Third Edition, Trinity University, (2005). 1

[4] E. M. Elsayed, A. Alotaibi, H. A. Almaylabi, On the periodicity and solutions of some difference equations systems, J. Comput. Theor. Nanosci., 13 (2016), 1624-1628. 1

[5] E. M. Elsayed, T. F. Ibrahim, Periodicity and solutions for some systems of nonlinear rational difference equations, Hacet. J. Math. Stat., 44 (2015), 1361-1390.

[6] E. M. Elsayed, F. Alzahrani, I. Abbas, N. H. Alotaibi, Solutions of some difference equations systems and periodicity, J. Adv. Math., 16 (2019), 8247-8261. 1

[7] E. M. Elsayed, H. El-Metwally, On the solutions of some nonlinear systems of difference equations, Adv. Difference Equ., 2013 (2013), 14 pages.

[8] T. W. Hungerford, Algebra, Springer-Verlag, New York, (1974). 1

[9] B. D. Iričanin, S. Stević, Some systems of nonlinear difference equations of higher order with periodic solutions, Dyn. Contin. Discrete Impuls. Syst. Ser. A Math. Anal., 13 (2006), 499-507. 1, 4

[10] M. R. S. Kulenovic, G. Ladas, Dynamics of second order rational difference equations, with open problems and conjectures, Chapman and Hall/CRC, (2001). 1

[11] R. C. Lyness, Notes 1581, Math. Gaz, 26 (1942), 62-62. 1

[12] R. C. Lyness, Notes 1847, Math. Gaz, 29 (1945), 231-233.

[13] R.C. Lyness, Notes 2952, Math. Gaz, 45 (1961), 207-209. 1

[14] H. Bao, Dynamical behavior of a system of second-order nonlinear difference equations, Int. J. Differ. Equ., 2015 (2015), 7 pages.

[15] C. J. Schinas, Invariants for difference equations and systems of difference equations of rational form, J. Math. Anal. Appl., 216 (1997), 164-179. 1

[16] S. Sternberg, Group theory and physics, Cambridge University Press, Cambridge, (1994). 1

[17] S.Stević, M. A. Alghamdi, D. A. Maturi, N. Shahzad, On the periodicity of some classes of systems of nonlinear difference equations, Abstr. Appl. Anal., 2014 (2014), 6 pages. 1 